\title{
The mixing time of the Thorp shuffle
}

\author{
BEN MORRIS*
}

\begin{abstract}
The Thorp shuffle is defined as follows. Cut the deck into two equal piles. Drop the first card from the left pile or the right pile according to the outcome of a fair coin flip; then drop from the other pile. Continue this way until both piles are empty. We show that the mixing time for the Thorp shuffle with $2^{d}$ cards is polynomial in $d$.
\end{abstract}

\section{Introduction}

\subsection{The Thorp shuffle}

How many shuffles are necessary to mix up a deck of cards? We refer to this as the mixing time (see section 1.2 for a precise definition). The mathematics of card shuffling has been studied extensively over the past several decades and most of the problems have been solved. Most famously, Bayer and Diaconis [1] (in one of the few mathematical results to have made the front page of the New York Times) gave very precise bounds for the Gilbert-Shannon-Reeds (riffle) shuffle model. Their bounds were correct even up to the constant factors. For almost all natural shuffles matching upper and lower bounds are known (often even up to constants). However, one card shuffling problem has stood out for its resistance to attack.

In 1973, Thorp [10] introduced the following shuffling procedure. Assume that the number of cards, $n$, is even. Cut the deck into two equal piles. Drop the first card from the left pile or the right pile according to the outcome of a fair coin flip; then drop from the other pile. Continue this way, with independent coin flips deciding whether to drop LEFT-RIGHT or RIGHT-LEFT each time, until both piles are empty.

The Thorp shuffle, despite its simple description, has been hard to analyze. The problem of determining its mixing time is, according to Persi Diaconis [3], the "longest-standing open card shuffling problem." It has long been conjectured that the mixing time is $O\left(\log ^{c} n\right)$ for some constant c. However, despite much effort the only known upper bounds are trivial ones of the form $O\left(n^{c}\right)$ that have circulated in the folklore. The main contribution of this paper is to give the first poly log upper bound for the mixing time.

We shall assume that the number of cards is $2^{d}$ for a positive integer $d$. (Thus, our aim is to prove that the mixing time is polynomial in $d$.) In this case the Thorp shuffle has a very appealing alternative description. By writing the position of each card, from the bottom card (0) to the top card $\left(2^{d}-1\right)$, in binary, we can view the cards as occupying the vertices of the $d$-dimensional unit hypercube $\{0,1\}^{d}$. The Thorp shuffle proceeds in two stages. In the first stage, an independent

\footnotetext{
${ }^{*}$ Department of Mathematics, University of California, Davis. Email: morris@math.ucdavis.edu. This work was done while the author was at Indiana University and Microsoft Research.
} 
coin is flipped for each edge $e$ in direction 1 (i.e., each edge in the cube that connects two vertices that differ in only the first coordinate). If the coin lands heads, the cards at the endpoints of $e$ are interchanged; otherwise the cards remain in place. In the second stage, a "cyclic left bit shift" is performed for each card, where the card in position $\left(x_{1}, \ldots, x_{d}\right)$ is moved to $\left(x_{2}, \ldots, x_{d}, x_{1}\right)$.

We will actually use a slightly modified definition of the Thorp shuffle. Say that an edge in the hypercube rings if its endpoints are switched with probability $\frac{1}{2}$. For $j=1, \ldots, d$, let $K_{j}$ be the transition kernel for the the process in which every edge $e$ in direction $j$ rings.

Definition: Thorp shuffle. The Thorp shuffle is the Markov chain whose transition kernel at time $n$ is $K_{j+1}$ if $j \equiv n \bmod d$.

Since $d$ iterations of this shuffle is equivalent to $d$ iterations of the shuffle described in [10], it is enough to prove a poly $(d)$ mixing time bound for this new model.

It is natural to consider the change in the deck after $d$ shuffles have been performed. (This represents one complete "cycle".) We will call this a round. Using the language of network computing, a round of the Thorp shuffle is like passing the cards through $d$ levels of a butterfly network (see, e.g., Knuth's book [6]), where at each stage neighboring cards are interchanged with probability $\frac{1}{2}$. We note that in a recent breakthrough result, Çam [2] showed that the matrix $K_{1} \cdots K_{d} K_{1} \cdots K_{d-1}$ has strictly positive entries. This can be viewed as a result about the "diameter" of the Thorp shuffle; after a small number of steps there is a positive probability of being in any given state. However, these probabilities are in general very small so this does not imply a good bound for the mixing time.

The main result of this paper is that indeed the mixing time is polynomial in $d$. Our proof uses evolving sets, a technique for bounding mixing times that was introduced by the author and Peres in [8. Another paper that uses some of the same ideas is [7], in which a variant of evolving sets is used to analyze the exclusion process. Evolving sets are related to the notion of strong stationary duality due to Diaconis and Fill [5].

\subsection{Statement of main result}

For a Markov chain on state space $V$ with uniform stationary distribution, define the (uniform) mixing time by

$$
\tau_{\text {mix }}=\min \left\{n:\left|p^{n}(x, y)\right| V|-1| \leq \frac{1}{4} \forall x, y \in V\right\},
$$

where $p^{n}(x, y)$ is the $n$-step transition probability from $x$ to $y$. (This is a stricter definition of mixing time than the usual one involving total variation distance.)

Our main result is the following theorem.

Theorem 1 The mixing time for the Thorp shuffle is $O\left(d^{44}\right)$.

In similar fashion to the analysis in [8, we prove our mixing time bound based on an isoperimetric function we call the root profile. The paper is organized as follows. Following a brief introduction to evolving sets in section [2] we devote much of the rest of the paper to proving a bound on the root profile. In section 3 we show how $l^{2}$ techniques can be combined with evolving sets to give a bound on the root profile. In section 4 we describe the chameleon process, a variant of the Thorp shuffle in which the cards have changing colors, which is useful to bound mixing times. In section [5 we use the chameleon process to show that for a "reversibilized" version of the Thorp shuffle, any collection of cards (if viewed as indistinguishable) mixes in poly $(d)$ time. In section [6 we state the main technical result of this paper (proved in section 9), which says that the transition 
kernel for the Thorp shuffle contracts functions in a certain $l^{2}$ sense; then we use this to obtain our bound on the root profile. Next, armed with a good bound on the root profile we prove Theorem [1 in section [7. We conclude with proofs of some technical lemmas in sections and 8 and 9 .

\section{Evolving sets}

We will now give a brief overview of evolving sets (see 8 for a more detailed account). Let $\{p(x, y)\}$ be transition probabilities for an irreducible, aperiodic Markov chain on a finite state space $V$. Assume that the chain has a uniform stationary distribution (which means that $p$ is doubly stochastic: $\sum_{x \in V} p(x, y)=1$ for all $\left.y \in V\right)$. For subsets $S \subset V$, define $p(S, y):=\sum_{x \in S} p(x, y)$.

Definition: Evolving sets. The evolving set process is the Markov chain $\left\{S_{n}\right\}$ on subsets of $V$ with the following transition rule. If the current state $S_{n}$ is $S \subset V$, choose $U$ uniformly from $[0,1]$ and let the next state $S_{n+1}$ be

$$
\widetilde{S}=\{y: p(S, y) \geq U\} .
$$

Write $\mathbf{P}_{S}(\cdot):=\mathbf{P}\left(\cdot \mid S_{0}=S\right)$ and similarly for $\mathbf{E}_{S}(\cdot)$. Evolving sets have the following properties (see [8]).

1. The sequence $\left\{\left|S_{n}\right|\right\}_{n \geq 0}$ forms a martingale.

2. For all $n \geq 0$ and $x, y \in V$ we have

$$
p^{n}(x, y)=\mathbf{P}_{\{x\}}\left(y \in S_{n}\right) .
$$

3. The sequence of complements $\left\{S_{n}^{c}\right\}_{n \geq 0}$ is also an evolving set process, with the same transition probabilities.

As in [8, we will prove our mixing time bound using an isoperimetric quantity that we denote by $\psi$, which is defined as follows. For $S \subset V$, define

$$
\psi(S):=1-\mathbf{E}_{S} \sqrt{\frac{|\widetilde{S}|}{|S|}} .
$$

Define $\psi(x)$ for $x \in[0,1 / 2]$ by

$$
\psi(x)=\inf \{\psi(S):|S| \leq x|V|\}
$$

and for $x>1 / 2$, let $\psi(x):=\psi_{*}=\psi\left(\frac{1}{2}\right)$. Observe that $\psi$ is non-negative and (weakly) decreasing on $[0, \infty)$. We will call the function $\psi$ the root profile.

\section{From $\ell^{2}$ bounds to a bound on $\psi$}

In this section, we show how to use $l^{2}$ techniques to obtain a bound on the root profile.

Let $p(x, y)$ be a doubly stochastic Markov chain on the state space $V$. For functions $f$ : $V \rightarrow[0,1]$, define $\|f\|_{1}:=\frac{1}{|V|} \sum_{x \in V} f(x)$ and $\|f\|_{2}:=\left(\frac{1}{|V|} \sum_{x \in V} f(x)^{2}\right)^{1 / 2}$. For $S \subset V$, define $\mathbf{1}_{S}: V \rightarrow[0,1]$ by

$$
\mathbf{1}_{S}(x)= \begin{cases}1 & \text { if } x \in S \\ 0 & \text { otherwise }\end{cases}
$$


Lemma 2 Let $\widetilde{S}$ be the next step in the evolving set process starting from $S$, i.e., $\widetilde{S}=\{y: p(S, y)>$ $U\}$, where $U$ is uniform. Let $\alpha=\frac{\|p(S, \cdot)\|_{2}^{2}}{\left\|\mathbf{1}_{S}\right\|_{1}}$. Then

$$
\mathbf{E}\left(\sqrt{\frac{|\widetilde{S}|}{|S|}}\right) \leq[\alpha(2-\alpha)]^{\frac{1}{4}} .
$$

Proof: Let $\Lambda$ be an independent copy of $\widetilde{S}$, i.e., $\Lambda=\left\{y: p(S, y)>U^{\prime}\right\}$, for an independent uniform random variable $U^{\prime}$. Note that either $\widetilde{S} \subseteq \Lambda$ or $\Lambda \subseteq \widetilde{S}$ (depending on which of the uniform variables $U, U^{\prime}$ is larger). Let $X=|\widetilde{S} \cap \Lambda|$ and $Y=|\widetilde{S} \cup \Lambda|$. Then

$$
\begin{aligned}
{[\mathbf{E}(\sqrt{|\widetilde{S}|})]^{2} } & =\mathbf{E}(\sqrt{|\widetilde{S}||\Lambda|}) \\
& =\mathbf{E}(\sqrt{X Y}) \\
& \leq \sqrt{\mathbf{E}(X) \mathbf{E}(Y)} \\
& =\sqrt{\mathbf{E}(X)(2|S|-\mathbf{E}(X))},
\end{aligned}
$$

where the first inequality is Cauchy Schwarz and the second inequality follows from the fact that $\mathbf{E}(X+Y)=2 \mathbf{E}(\widetilde{S})=2|S|$. But

$$
\begin{aligned}
\mathbf{E}(X) & =\sum_{y \in V} \mathbf{P}(y \in \widetilde{S} \cap \Lambda) \\
& =\sum_{y \in V} \mathbf{P}(y \in \widetilde{S})^{2} \\
& =\sum_{y \in V} p(S, y)^{2}=|V| \cdot\|p(S, \cdot)\|_{2}^{2},
\end{aligned}
$$

so dividing the LHS of (2) and the RHS of (15) by $|S|=|V| \cdot\left\|\mathbf{1}_{S}\right\|_{1}$ and then taking a square root yields the lemma.

Remark: The same proof shows that if $\widetilde{S}=\{y: f(y)>U\}$ for $f: V \rightarrow[0,1]$ arbitrary, then

$$
\mathbf{E}\left(\sqrt{\frac{\left\|\mathbf{1}_{\widetilde{S}}\right\|_{1}}{\|f\|_{1}}}\right) \leq[\alpha(2-\alpha)]^{\frac{1}{4}},
$$

where $\alpha=\frac{\left\|K^{t} f\right\|_{2}^{2}}{\|f\|_{1}}$ for $K$ the transition kernel. Note also that if we define $\Delta:=1-\alpha$, then

$$
[\alpha(2-\alpha)]^{\frac{1}{4}}=\left(1-\Delta^{2}\right)^{\frac{1}{4}} \leq 1-\frac{\Delta^{2}}{4}
$$




\section{Chameleon process}

It will be convenient to study the card shuffle that behaves like the Thorp shuffle for the first $d$ steps $\left(K_{1}, \ldots, K_{d}\right)$, and then like a "reverse Thorp shuffle" for the next $d$ steps $\left(K_{d}, \ldots, K_{1}\right)$. We will call this the zigzag shuffle. Every $2 d$ steps of the zigzag shuffle will be called a round. (So a round of the zigzag shuffle is a round of the Thorp shuffle followed by a round of a time-reversed Thorp shuffle.)

Let $\alpha$ be large enough so that $4 \alpha^{-d} \leq 2^{-d-1} 4^{-d}$ for all $d \geq 1$ and let $c$ be an integer large enough so that $\left[4 e^{-c}\right]^{d} \beta \log \alpha c d^{5} \leq \alpha^{-d}$ for all $d \geq 1$, where $\beta=2056 \cdot 64 \cdot 5$.

The chameleon process is an extension of the zigzag shuffle. The cards move in the same way as in the zigzag shuffle, but they also have colors, which can be red, white, black or pink. Initially, the cards are colored as follows. There is a sequence of cards $x_{1}, \ldots, x_{b}$ for some $b>2^{d-1}$ such that cards $x_{1}, \ldots, x_{b-1}$ are colored white, card $x_{b}$ is colored red, and the remaining cards are colored black. The cards can change color in two ways. The first way is called pinkening, which takes place when an edge connecting a red card to a white card rings; in this case both cards are re-colored pink. The second way is called de-pinking, which takes place at the end of every $64 c d$ rounds of shuffling; in this case all of the pink cards are collectively re-colored red or white, with probaility $\frac{1}{2}$ each. (A process of this type was first used in [7] to analyze the exclusion process.) Note that black cards can never change color.

Let $X_{n}$ be the zigzag shuffle. For $j=1, \ldots, 2^{d}$, we will write $X_{n}(j)$ for the position of card $j$ at time $n$. If $S=\left\{z_{1}, \ldots, z_{k}\right\}$ is a set of cards, define $X_{n}(S)=\left\{X_{n}\left(z_{1}\right), \ldots, X_{n}\left(z_{k}\right)\right\}$. Let $W_{n}=X_{n}(\{1, \ldots, b\})$ be the unordered set of locations of nonblack (i.e., white, red or pink) cards at time $n$. For vertices $x$ in the hypercube, define

$$
\rho_{n}(x)=\mathbf{1}(\text { there is a red card at } x \text { at time } n)+\frac{1}{2} \mathbf{1}(\text { there is a pink card at } x \text { at time } n) .
$$

The following lemma indicates the fundamental relationship between the chameleon process and the zigzag shuffle.

Lemma 3 Consider the chameleon process with $b$ nonblack cards. Then

$$
\mathbf{P}\left(X_{n}\left(x_{b}\right)=x \mid W_{1}, W_{2}, \ldots\right)=\mathbf{E}\left(\rho_{n}(x) \mid W_{1}, W_{2}, \ldots\right) .
$$

Proof: We will use induction on $n$. The base case $n=0$ is trivial because there is initially only one red ball which is located at the position of card $x_{b}$. Now assume that the result holds for $n$. Let $e$ be the edge incident to $x$ that rings at time $n$ and let $x^{\prime}$ be the neighbor of $x$ across $e$. Let $A_{1}, A_{2}$ and $A_{3}$

be the events corresponding to the following three possible values of $\left(W_{n} \cap\left\{x, x^{\prime}\right\}, W_{n+1} \cap\left\{x, x^{\prime}\right\}\right)$ when $x \in W_{n+1}$ :

1. $\left(\left\{x, x^{\prime}\right\},\left\{x, x^{\prime}\right\}\right)$;

2. $\left(\left\{x^{\prime}\right\},\{x\}\right)$;

3. $(\{x\},\{x\})$.

Let $\mathcal{F}_{n}=\sigma\left(\rho_{n}(x), \rho_{n}\left(x^{\prime}\right)\right)$. Note that

$$
\mathbf{E}\left(\rho_{n+1}(x) \mid \mathcal{F}_{n}, W_{1}, W_{2}, \ldots\right)=\left(\frac{1}{2} \rho_{n}(x)+\frac{1}{2} \rho_{n}\left(x^{\prime}\right)\right) \mathbf{1}\left(A_{1}\right)+\rho_{n}\left(x^{\prime}\right) \mathbf{1}\left(A_{2}\right)+\rho_{n}(x) \mathbf{1}\left(A_{3}\right) .
$$


Define $\mu_{n}(\cdot)=\mathbf{P}\left(X_{n}\left(x_{b}\right)=\cdot \mid W_{1}, W_{2}, \ldots\right)$. Then

$$
\mu_{n+1}(x)=\left(\frac{1}{2} \mu_{n}(x)+\frac{1}{2} \mu_{n}\left(x^{\prime}\right)\right) \mathbf{1}\left(A_{1}\right)+\mu_{n}\left(x^{\prime}\right) \mathbf{1}\left(A_{2}\right)+\mu_{n}(x) \mathbf{1}\left(A_{3}\right) .
$$

But by induction we have

$$
\mu_{n}(x)=\mathbf{E}\left(\rho_{n}(x) \mid W_{1}, W_{2}, \ldots\right) ; \quad \mu_{n}\left(x^{\prime}\right)=\mathbf{E}\left(\rho_{n}\left(x^{\prime}\right) \mid W_{1}, W_{2}, \ldots\right) .
$$

To complete the proof, take the conditional expectation given $W_{1}, W_{2}, \ldots$ of both sides of (9) and combine with equation (10).

Remark: Note that

$$
\mathbf{E}\left(\sum_{x} \rho_{n}(x) \mid W_{1}, W_{2}, \ldots\right)=\sum_{x} \mathbf{P}\left(X_{n}(b)=x \mid W_{1}, W_{2}, \ldots\right)=1 .
$$

\section{Indistinguishable cards mix in poly time}

Let $\Lambda$ be a set of cards. Then the process $\left\{X_{n}(\Lambda): n \geq 0\right\}$ is a Markov chain. The following lemma says that the uniform mixing time for this chain is $O\left(d^{5}\right)$.

Lemma 4 There is a universal constant $b \in \mathbf{Z}$ such that if $m=b d^{5}$ then

$$
\max _{\Lambda, \Lambda^{\prime}}\left|\left(\begin{array}{l}
2^{d} \\
|\Lambda|
\end{array}\right) \mathbf{P}\left(\Lambda \rightarrow_{m} \Lambda^{\prime}\right)-1\right| \leq \frac{1}{4},
$$

where we write $\Lambda \rightarrow_{m} \Lambda^{\prime}$ for the event that $X_{m}(\Lambda)=\Lambda^{\prime}$.

Proof: It is enough to consider sets $\Lambda$ with $|\Lambda| \geq 2^{d-1}$. (Otherwise, consider $\Lambda^{c}$.) Let $\alpha, \beta$ and $c$ be defined as in section 4, let $b \geq \beta \log \alpha c$, and let $m=b d^{5}$. For $j \in\left\{1, \ldots, 2^{d}\right\}$ define

$$
\lambda(j)=\max _{|S|=j} \max _{\left|S^{\prime}\right|=j}\left|\left(2^{d}\right) \mathbf{P}\left(S \rightarrow_{m} S^{\prime}\right)-1\right| .
$$

We will show that for all $k \geq 2^{d-1}$, we have

$$
\lambda(k) \leq k^{\star} 4^{-d},
$$

where $k^{\star}=2^{d}-k$. This yields the lemma because the r.h.s. of (13) is at most $\frac{1}{4}$ for all $d \geq 1$.

Let $A$ and $B$ be disjoint sets of cards. For $x \in A$, say that $x$ is antisocial in round $j$ of the zigzag shuffle if at no point in round $j$ does an edge connecting $x$ to a card in $B$ ring. Let $Z(A, B, j)$ denote the number of cards that are antisocial in round $j$. We say that $A$ avoids $B$ if $Z(A, B, j)>\frac{7}{8}|A|$ for $64 c d$ consecutive rounds $j$ before time $m$. If $S$ is a set of cards, say that $S$ mixes if there do not exist disjoint sets $A, B$ of cards with $|A| \leq \frac{1}{2}|S|$ and $A \cup B=S$ such that $A$ avoids $B$.

We will verify (13) by induction on $k^{\star}$. The base case $k^{\star}=0\left(k=2^{d}\right)$ is trivial. Suppose it's true for $k$, where $k>2^{d-1}$ and consider $k-1$. Fix a set of cards $S=\left\{x_{1}, \ldots, x_{k}\right\}$ and consider the corresponding chameleon process. Let $\mathcal{F}=\sigma\left(X_{n}(S): n \geq 0\right)$. Let $Z_{n}=\sum_{x} \rho_{64 c d^{2} n}(x)$ be the 
total amount of "red paint" in the system after $64 c d n$ rounds of the chameleon process. Define $Z_{n}^{\sharp}=\min \left(Z_{n}, k-Z_{n}\right)$. Note that $\lim _{n \rightarrow \infty} Z_{n}^{\sharp}=0$ a.s.

Fix $n$ such that $64 c d^{2} n \leq m$, and let $A_{n}$ be either the set of cards that are red or the set of cards that are white at the start of round $64 c d n$, according to whether $Z_{n} \leq k / 2$ or $Z_{n}>k / 2$, respectively. Let $P$ denote the number of cards pinkened during the next $64 c d$ rounds. Let $B_{n}=S-A_{n}$. When $S$ mixes, $A_{n}$ doesn't avoid $B_{n}$. We claim that this ensures that $P \geq \frac{\left|A_{n}\right|}{8 d}$. Consider a round $j$ such that $Z\left(A_{n}, B_{n}, j\right) \geq \frac{7}{8}\left|A_{n}\right|$. Note that after an edge connecting a card $x$ in $A_{n}$ to a card $y$ in $B_{n}$ rings, at least one of the resulting cards is pink. Let us associate that pink card with $x$. (If both endpoints are pink then choose one of them arbitrarily.) Since at least a fraction $1 / 8$ of the cards in $A_{n}$ will have a pink card associated to them in this round, and since any given pink card can be associated to at most $d$ cards in $A_{n}$ in this round, the number of pink cards at the end of this round must be at least $\frac{\left|A_{n}\right|}{8 d}$. It follows that $P \geq \frac{\left|A_{n}\right|}{8 d}$.

Note that $Z_{n+1}$ is either $Z_{n}+\frac{1}{2} P$ or $Z_{n}-\frac{1}{2} P$, with probability $\frac{1}{2}$ each. Thus, if we write $E$ for the event that $S$ does not mix, then

$$
\begin{aligned}
\mathbf{E}\left(\sqrt{Z_{n+1}^{\sharp}} \mid P, Z_{n}, \mathcal{F}, E^{c}\right) & =\mathbf{E}\left(\frac{1}{2} \sqrt{\left(Z_{n}+\frac{1}{2} P\right)^{\sharp}}+\frac{1}{2} \sqrt{\left(Z_{n}-\frac{1}{2} P\right)^{\sharp}} \mid Z_{n}, \mathcal{F}, E^{c}\right) \\
& \leq \sqrt{Z_{n}^{\sharp}} \frac{\sqrt{1+\frac{1}{16 d}}+\sqrt{1-\frac{1}{16 d}}}{2} \\
& \leq \sqrt{Z_{n}^{\sharp}} \exp \left[-\frac{1}{2056 d^{2}}\right],
\end{aligned}
$$

where the first inequality follows from the concavity of the square root, and the second inequality follows from the fact that $\frac{1}{2} \sqrt{1+u}+\frac{1}{2} \sqrt{1-u} \leq \exp \left(-u^{2} / 8\right)$ whenever $u \in[0,1]$ (see [8], Lemma 9).

Thus, since $Z_{0}=1$, it follows that

$$
\mathbf{E}\left(\sqrt{Z_{n}^{\sharp}} \mid \mathcal{F}, S \text { mixes }\right) \leq \exp \left[-\frac{n}{2056 d^{2}}\right]
$$

for all $n$. Define $Z_{\infty}=\lim _{n \rightarrow \infty} Z_{n}$. (Note that for any $S^{\prime}$ we have $\mathbf{E}\left(Z_{\infty} \mid S \rightarrow_{m} S^{\prime}\right)=1$; see the remark immediately following Lemma 3]) Lemma 3 implies that for all $y \in S^{\prime}$ we have

$$
\begin{aligned}
\left|\mathbf{P}\left(X_{m}\left(x_{k}\right)=y \mid S \rightarrow_{m} S^{\prime}\right)-\frac{1}{k}\right| & =\left|\mathbf{E}\left(\rho_{m}(y)-\frac{1}{k} Z_{\infty} \mid S \rightarrow_{m} S^{\prime}\right)\right| \\
& \leq \mathbf{E}\left(\left|\rho_{m}(y)-\frac{1}{k} Z_{\infty}\right| \mid S \rightarrow_{m} S^{\prime}\right) \\
& \leq \mathbf{P}\left(\rho_{m} \notin\{0, k\} \mid S \rightarrow_{m} S^{\prime}\right) .
\end{aligned}
$$

Let $E$ be the event that $S$ does not mix. Lemma 9 in Appendix A gives $\mathbf{P}\left(E \mid S \rightarrow_{m} S^{\prime}\right) \leq \alpha^{-d} \frac{1+\lambda(k)}{1-\lambda(k)}$. Hence

$$
\begin{aligned}
\mathbf{P}\left(\rho_{m} \notin\{0, k\} \mid S \rightarrow_{m} S^{\prime}\right) & \leq \mathbf{P}\left(E \mid S \rightarrow_{m} S^{\prime}\right)+\mathbf{P}\left(\rho_{m} \notin\{0, k\} \mid S \rightarrow_{m} S^{\prime}, E^{c}\right) \\
& \leq \alpha^{-d} \frac{1+\lambda(k)}{1-\lambda(k)}+\mathbf{P}\left(\rho_{m} \notin\{0, k\} \mid S \rightarrow_{m} S^{\prime}, E^{c}\right) \\
& \leq 3 \alpha^{-d}+\mathbf{P}\left(\rho_{m} \notin\{0, k\} \mid S \rightarrow_{m} S^{\prime}, E^{c}\right)
\end{aligned}
$$


where the third inequality holds because $\lambda(k) \leq \frac{1}{4}$ by induction. But

$$
\begin{aligned}
\mathbf{P}\left(\rho_{m} \notin\{0, k\} \mid S \rightarrow_{m} S^{\prime}, E^{c}\right) & \leq \mathbf{E}\left(Z_{m / 64 c d^{2}}^{\sharp} \mid S \rightarrow_{m} S^{\prime}, E^{c}\right) \\
& \leq \exp \left[-\frac{m}{2056 \cdot 64 \cdot c d^{4}}\right] \leq \alpha^{-d},
\end{aligned}
$$

where the second inequality follows from equation (17). Combining equations (20), (23), and (25) gives

$$
\left|\mathbf{P}\left(X_{m}\left(x_{k}\right)=y \mid S \rightarrow_{m} S^{\prime}\right)-\frac{1}{k}\right| \leq 4 \alpha^{-d} .
$$

Now fix a set of cards $\Lambda$ with $|\Lambda|=k-1$ and and let $z \notin \Lambda$. Define $\Lambda_{z}=\Lambda \cup z$. Fix a set $\Lambda^{\prime}$ of vertices of the hypercube with $\left|\Lambda^{\prime}\right|=k-1$. For $w \notin \Lambda^{\prime}$, define

$$
\begin{array}{rr}
x_{w}=\mathbf{P}\left(\Lambda_{z} \rightarrow_{m} \Lambda_{w}^{\prime}\right) & \Delta x_{w}=x_{w}-\left(\begin{array}{c}
2^{d} \\
k
\end{array}\right) \\
y_{w}=\mathbf{P}\left(z \rightarrow_{m} w \mid \Lambda_{z} \rightarrow_{m} \Lambda_{w}^{\prime}\right) & \Delta y_{w}=y_{w}-1 / k .
\end{array}
$$

Note that $\left|\left\{w: w \notin \Lambda^{\prime}\right\}\right|=k^{\star}+1$, and $\frac{k^{\star}+1}{k}\left(\begin{array}{c}2^{d} \\ k\end{array}\right)^{-1}=\left(\begin{array}{c}2^{d} \\ k-1\end{array}\right)^{-1}$. It follows that

$$
\begin{aligned}
\left|\mathbf{P}\left(\Lambda \rightarrow_{m} \Lambda^{\prime}\right)-\left(\begin{array}{c}
2^{d} \\
k-1
\end{array}\right){ }^{-1}\right| & =\left|\sum_{w \notin \Lambda^{\prime}} \mathbf{P}\left(\Lambda_{z} \rightarrow_{m} \Lambda_{w}^{\prime}, z \rightarrow_{m} w\right)-\frac{1}{k}\left(\begin{array}{c}
2^{d} \\
k
\end{array}\right){ }^{-1}\right| \\
& =\left|\sum_{w \notin \Lambda^{\prime}} x_{w} y_{w}-\frac{1}{k}\left(\begin{array}{c}
2^{d} \\
k
\end{array}\right)\right| \\
& =\left|\sum_{w \notin \Lambda^{\prime}} \Delta x_{w} \frac{1}{k}+\Delta y_{w}\left(\begin{array}{c}
2^{d} \\
k
\end{array}\right)+\Delta x_{w} \Delta y_{w}\right| .
\end{aligned}
$$

Note that

$$
\left|\Delta x_{w}\right| \leq k^{\star} 4^{-d}\left(\begin{array}{c}
2^{d} \\
k
\end{array}\right)^{-1} \leq\left(\begin{array}{c}
2^{d} \\
k
\end{array}\right)^{-1}
$$

where the first inequality is induction and the second inequality holds because $k^{\star} \leq 2^{d}$. Also, equation (26) implies that

$$
\left|\Delta y_{w}\right| \leq 4 \alpha^{-d} \leq \frac{1}{2 k} 4^{-d}
$$

for all $d \geq 1$ by the definition of $\alpha$. Thus, using equations (32), (33) and the triangle inequality, equation (31) becomes

$$
\begin{aligned}
\mid \mathbf{P}\left(\Lambda \rightarrow_{m} \Lambda^{\prime}\right)-\left(\begin{array}{c}
2^{d} \\
k-1
\end{array}\right) & -1 \\
& \leq \frac{k^{\star}+1}{k}\left[k^{\star} 4^{-d}\left(\begin{array}{c}
2^{d} \\
k
\end{array}\right)+4^{-d}\left(\begin{array}{c}
2^{d} \\
k
\end{array}\right)\right] \\
& =\frac{1}{k}\left(k^{\star}+1\right)^{2} 4^{-d}\left(\begin{array}{c}
2^{d} \\
k
\end{array}\right) \\
& =\left(k^{\star}+1\right) 4^{-d}\left(\begin{array}{c}
2^{d} \\
k-1
\end{array}\right)^{-1}=(k-1)^{\star} 4^{-d}\left(\begin{array}{c}
2^{d} \\
k-1
\end{array}\right)
\end{aligned}
$$

Since this is true for all $\Lambda$ with $|\Lambda|=k-1$ the proof is complete. 
Let $K$ be the transition kernel for one round of the Thorp shuffle, and let $K^{t}$ be the transpose of $K$, defined by $K^{t}(x, y)=K(y, x)$. Note that $K^{t}$ is the time-reversal of $K$. Let $\hat{K}:=K K^{t}$ be the transition kernel for one round of the zigzag shuffle. Let $\left\{Z_{n}: n \geq 0\right\}$ be a Markov chain with transition kernel $\hat{K}$. Then Lemma $[$ implies that for any set of cards $B$, the uniform mixing time for the process $\left\{Z_{n}(B): n \geq 0\right\}$ is at most $b d^{4}$. Thus, using standard facts about geometric convergence and the uniform mixing time, we can conclude that for a universal constant $C$ we have

$$
\max _{B^{\prime}}\left(\begin{array}{c}
2^{d} \\
|B|
\end{array}\right) \mathbf{P}\left(Z_{k C d^{4}}(B)=B^{\prime}\right) \leq 1+e^{-k}
$$

for all $k \geq 1$.

Truncated Thorp shuffle. Fix $d_{\star} \leq d$. Define the $d_{\star}$-truncated Thorp shuffle as the Markov chain with transition kernel $K_{\star}=K_{1} \ldots K_{d_{\star}}$. This is a "partial round" of the Thorp shuffle, with steps $d_{\star}+1$ through $d$ censored. To make things irreducible, we define the state space as the set of states reachable from an (arbitrary) fixed starting state.

Define the $d_{\star}$-truncated zigzag shuffle as the Markov chain with transition kernel $K_{\star} K_{\star}^{t}$. Note that we can think of this shuffle as a product of $2^{d-d_{\star}}$ copies of a " $d_{\star}$-dimensional" zigzag shuffle, where the cards occupy $2^{d-d_{\star}}$ (disconnected) hypercubes of dimension $d_{\star}$. Combining this observation with equation (34) yields the following corollary to Lemma 5 .

Corollary 5 Fix $d_{\star} \geq 2$ and let $\left\{Z_{n}: n \geq 0\right\}$ be the $d_{\star}$-truncated zigzag shuffle. There is a universal constant $c$ such that if $l=k c d\left(d_{\star}-1\right)^{4}$, then

$$
\max _{B^{\prime}}\left(\begin{array}{c}
2^{d} \\
|B|
\end{array}\right) \mathbf{P}\left(Z_{l}(B)=B^{\prime}\right) \leq \exp (\exp (-k))
$$

for all $k \geq 1$.

Proof: Let $c=2^{5} C$. Then $l \geq 2 k d C d_{\star}^{4}$, so equation (34) implies that

$$
\begin{aligned}
\max _{B^{\prime}}\left(\begin{array}{c}
2^{d} \\
|B|
\end{array}\right) \mathbf{P}\left(Z_{l}(B)=B^{\prime}\right) & \leq\left(1+e^{-2 k d}\right)^{2^{d-d_{\star}}} \\
& \leq \exp \left(2^{d} \exp (-2 d k)\right) \\
& \leq \exp (\exp (-k))
\end{aligned}
$$

for all $d \geq 1$.

\section{A bound on the root profile}

We will need the following technical result, which is proved in Appendix B.

Corollary 13 Fix $S \subset V$ and let $x=\frac{|S|}{\left(2^{d}\right) !}=\left\|\mathbf{1}_{S}\right\|_{1}$. Let $p(\cdot, \cdot)$ be the transition kernel for one round of the Thorp shuffle. Then there is a universal constant $C>0$ such that

$$
\|p(S, \cdot)\|_{2}^{2} \leq x^{1+C / d^{14}} .
$$

We are now ready to obtain a bound on the root profile of the Thorp shuffle. 
Lemma 6 Let $\psi$ be the root profile of the Markov chain which each step performs a round of the Thorp shuffle $\left(K_{1} K_{2} \cdots K_{d}\right)$. There is a universal constant $c>0$ such that

$$
\psi(x) \geq \max \left(1-x^{c / 2 d^{42}}, c d^{-28}\right) .
$$

Proof: Let $C$ be the constant appearing in Corollary 13. We will show that there is a universal constant $B>0$ such that

$$
\psi(x) \geq \max \left(1-x^{C B / 2 d^{42}}, B d^{-28}\right) .
$$

Setting $c=\min (B C, C)$ will then yield the lemma. First, we show that $\psi_{*} \geq B d^{-28}$. Fix $S$ with $\frac{|S|}{\left(2^{d}\right) !}=x \leq \frac{1}{2}$ and let

$$
\widetilde{S}=\{y: p(S, y)>U\}
$$

where $\{p(x, y)\}$ are the transition probabilities for one round of the Thorp shuffle. The remark following Lemma 2 implies that

$$
\mathbf{E} \sqrt{\frac{|\widetilde{S} \sharp|}{\left|S^{\sharp}\right|}} \leq 1-\frac{\Delta^{2}}{4},
$$

where $\Delta=1-\frac{\|p(S, \cdot)\|_{2}^{2}}{\left\|\mathbf{1}_{S}\right\|_{1}}$, and Corollary 13 implies that $\|p(S, \cdot)\|\left\|_{2}^{2} \leq x^{C / d^{14}}\right\| \mathbf{1}_{S}\left\|_{1} \leq 2^{-C / d^{14}}\right\| \mathbf{1}_{S} \|_{1}$. Thus

$$
\begin{aligned}
\Delta & \geq 1-2^{-C d^{-14}} \\
& =1-e^{-C \log 2 d^{-14}} \\
& \geq A d^{-14},
\end{aligned}
$$

for a universal constant $A>0$, and hence $1-\frac{\Delta^{2}}{4} \leq 1-B d^{-28}$ for a universal constant $B \in\left(0, \frac{1}{4}\right)$. (The fact that we can take $B<\frac{1}{4}$ will be used later on.) Since this holds for all $S$ with $|S| \leq \frac{1}{2}\left(2^{d}\right)$ !, we conclude that $\psi_{*} \geq B d^{-28}$. To complete the proof of Lemma 6] we must show that equation (37) holds when the max is achieved by the first term. Suppose that $1-x^{C B / 2 d^{42}} \geq B d^{-28}$. Then

$$
x \leq\left(1-B d^{-28}\right)^{2 d^{42} / C B} \leq \exp \left(-2 C^{-1} d^{14}\right) .
$$

Assume that (41) holds. Lemma 2 gives

$$
\mathbf{E} \sqrt{\frac{\left|\widetilde{S}^{\sharp}\right|}{\left|S^{\sharp}\right|}} \leq(\alpha(2-\alpha))^{\frac{1}{4}} \leq(2 \alpha)^{\frac{1}{4}},
$$

where $\alpha=\frac{\|p(S, \cdot)\|_{2}^{2}}{\left\|\mathbf{1}_{S}\right\|_{1}}$. Equation (41) implies that

$$
x^{C / 2 d^{14}} \leq e^{-1}<\frac{1}{2},
$$

and hence

$$
2 \leq x^{-C / 2 d^{14}} .
$$

Furthermore, Corollary 13 implies that $\alpha \leq x^{C / d^{14}}$. Plugging this and (43) into (42) gives

$$
\mathbf{E} \sqrt{\frac{\left|\widetilde{S}^{\sharp}\right|}{\left|S^{\sharp}\right|}} \leq\left(x^{-C / 2 d^{14}} x^{C / d^{14}}\right)^{\frac{1}{4}}=x^{C / 8 d^{14}} \leq x^{C B / 2 d^{42}},
$$

since $B<\frac{1}{4}($ and $x \leq 1)$. 


\section{Proof of main result}

Proof of Theorem [1 We shall start by bounding the mixing time of the Markov chain that does an entire round of the Thorp shuffle each step. Recall that the root profile $\psi:[0, \infty) \rightarrow \mathbf{R}$ is defined by

$$
\psi(x)= \begin{cases}\inf \{\psi(S):|S| \leq x|V|\} & \text { if } x \in\left[0, \frac{1}{2}\right] \\ \psi_{*} & \text { if } x>\frac{1}{2}\end{cases}
$$

where $\psi_{*}=\psi\left(\frac{1}{2}\right)$. Thus $\psi$ is (weakly) decreasing on $[0, \infty)$.

Let $h(z):=1-\psi\left(1 / z^{2}\right)$. Since $\psi(x)=\psi_{*}$ for all real numbers $x \geq \frac{1}{2}$, the function $h$ is welldefined even for $z \leq 1$. Note that $h$ is nonincreasing. In $[8$ it is shown (see section 5 and the part of section 3 entitled "Derivation of Theorem 1 from Lemma 3 and Theorem 4") that there is a sequence of random variables $\left\{Z_{n}: n \geq 0\right\}$ that satisfies $Z_{0}=\sqrt{|V|}$ and

$$
\mathbf{E}\left(\frac{Z_{n+1}}{Z_{n}} \mid Z_{n}\right) \leq h\left(Z_{n}\right)
$$

such that

$$
\tau_{\text {mix }} \leq 2 \min \left\{n: \mathbf{E}\left(Z_{n}\right) \leq \frac{1}{2}\right\} .
$$

Lemma 6 gave the following bound on the root profile:

$$
\psi(x) \geq \max \left(1-x^{c / 2 d^{42}}, c d^{-28}\right),
$$

for a universal constant $c>0$. Thus $h \leq g$, where $g$ is defined by

$$
g(z)=\min \left(z^{-c / d^{42}}, 1-c d^{-28}\right),
$$

and hence $\mathbf{E}\left(Z_{n+1} \mid Z_{n}\right) \leq g\left(Z_{n}\right) Z_{n}$. Let $f(z)=z g(z)=\min \left(z^{1-c / d^{42}}, z\left(1-c d^{-28}\right)\right)$. Note that $f$ is increasing and, as the minimum of two concave functions, is concave. We claim that $\mathbf{E}\left(Z_{n}\right) \leq$ $f^{n}\left(Z_{0}\right)$, where $f^{n}$ is the $n$-fold iterate of $f$. We verify this by induction. The base case $n=0$ is immediate. Suppose that the claim holds for $n$. Then

$$
\begin{aligned}
\mathbf{E}\left(Z_{n+1}\right) & =\mathbf{E}\left(\mathbf{E}\left(Z_{n+1} \mid Z_{n}\right)\right) \\
& \leq \mathbf{E}\left(f\left(Z_{n}\right)\right) \\
& \leq f\left(\mathbf{E}\left(Z_{n}\right)\right) \\
& \leq f\left(f^{n}\left(Z_{0}\right)\right)=f^{n+1}\left(Z_{0}\right),
\end{aligned}
$$

where the third line follows from concavity and the last line is the induction hypothesis. Let

$$
f_{1}=z^{1-c / d^{42}} ; \quad f_{2}=z\left(1-c d^{-28}\right),
$$

so that $f=\min \left(f_{1}, f_{2}\right)$. Then for all $m, n$ we have

$$
\mathbf{E}\left(Z_{m+n}\right) \leq f^{m+n}\left(\sqrt{Z_{0}}\right) \leq f_{2}^{m}\left(f_{1}^{n}\left(Z_{0}\right)\right)
$$

But $f_{1}^{n}(z)=z^{\left(1-c / d^{42}\right)^{n}} \leq z^{\exp \left(-c n / d^{42}\right)}$, and $Z_{0}=\sqrt{|V|} \leq\left(2^{d}\right)^{2^{d}}=2^{d 2^{d}}$. Thus, choosing $n \geq c^{-1} d^{43}$ gives

$$
f_{1}^{n}\left(Z_{0}\right) \leq 2^{d 2^{d} e^{-d}}
$$


which is at most 4 for all $d \geq 1$. Finally, since

$$
f_{2}^{m}(z)=z\left(1-c d^{-28}\right)^{m} \leq z e^{-c m / d^{28}},
$$

we have $f_{2}^{m}(4) \leq 4 e^{-c m / d^{28}}$, which is at most $\frac{1}{2}$ whenever $m \geq c^{-1} d^{28} \log 8$. Putting this together, we conclude that $\tau_{\text {mix }} \leq 2 c^{-1}\left(d^{43}+d^{28} \log 8\right)=O\left(d^{43}\right)$. Since each round corresponds to $d$ Thorp shuffles we conclude that the mixing time for the original model is $O\left(d^{44}\right)$.

\section{Appendix A}

In this section we prove some large deviation results needed in section [5. We will adopt the notation of that section; for the convenience of the reader, we now give a brief recap. Let $A$ and $B$ be disjoint sets of cards. For $x \in A$, say that $x$ is antisocial in round $j$ of the zigzag shuffle if at no point in round $j$ does an edge connecting $x$ to a card in $B$ ring. Let $Z(A, B, j)$ denote the number of cards that are antisocial in round $j$. We say that $A$ avoids $B$ if $Z(A, B, j)>\frac{7}{8}|A|$ for $64 c d$ consecutive rounds $j$ before time $m$. If $S$ is a set of cards, say that $S$ mixes if there do not exist disjoint sets $A, B$ of cards with $|A| \leq \frac{1}{2}|S|$ and $A \cup B=S$ such that $A$ avoids $B$.

Lemma 7 Let $\left\{X_{n}: n \geq 0\right\}$ be the zigzag shuffle. Let $Z=Z(A, B, 1)$ be the number of cards that are antisocial in the first round. Define $\mathcal{F}_{B}=\sigma\left(X_{1}(B), \ldots, X_{d}(B)\right)$. Let $p=1-\frac{|B|}{2^{d}}$ and let $k=|A|$. For $\theta \geq 0$ define $\Phi_{p}(\theta)=1-p+p e^{\theta}$. Then for all $\theta \geq 0$ we have

$$
\mathbf{E}\left(e^{\theta Z} \mid \mathcal{F}_{B}\right) \leq \Phi_{p}(\theta)^{k}
$$

Proof: We verify this by induction on $d$. If $d=1$ then the LHS of (52) is 1 if $p<1$, and $e^{\theta k}$ otherwise, so (52) holds. Now suppose that $d>1$. Let $A^{\prime}$ be the set of cards in $A$ not adjacent to $B$ in direction 1 , and let $k^{\prime}=\left|A^{\prime}\right|$. Let $l$ be half the number of cards in $A^{\prime}$ adjacent to another card in $A^{\prime}$ in direction 1 . (Note that $l$ is an integer.) Let $k_{0}$ and $k_{1}$ be the number of cards in $A^{\prime}$ that end up with a leading 0 and 1 , respectively, after the first step of the round (i.e., after the edges in direction $1 \mathrm{ring}$ ). Of those in the first group, let $Z_{0}$ be the number that are antisocial, with a similar definition for $Z_{1}$. Note that given $\mathcal{F}_{B}$, the random variables $k_{0}$ and $k_{1}$ are both distributed like $W+l$, where $W \sim \operatorname{Binomial}\left(k^{\prime}-2 l, \frac{1}{2}\right)$, and note that $Z=Z_{0}+Z_{1}$. By induction, we have

$$
\begin{aligned}
\mathbf{E}\left(e^{\theta Z} \mid \mathcal{F}_{B}, X_{1}(A)\right) & =\mathbf{E}\left(e^{\theta Z_{0}} \mid \mathcal{F}_{B}, X_{1}(A)\right) \mathbf{E}\left(e^{\theta Z_{1}} \mid \mathcal{F}_{B}, X_{1}(A)\right) \\
& \leq \Phi_{p_{0}}(\theta)^{k_{0}} \Phi_{p_{1}}(\theta)^{k_{1}},
\end{aligned}
$$

where $p_{0}$ is the fraction of locations of the part of the hypercube with a leading 0 not occupied by a card in $B$ after the first step, with a similar definition for $p_{1}$. It follows that $\mathbf{E}\left(e^{\theta Z} \mid \mathcal{F}_{B}, k_{0}, k_{1}\right) \leq$ $\Phi_{p_{0}}(\theta)^{k_{0}} \Phi_{p_{1}}(\theta)^{k_{1}}$. Hence

$$
\begin{aligned}
\mathbf{E}\left(e^{\theta Z} \mid \mathcal{F}_{B}\right) & \leq \sum_{i=0}^{k^{\prime}-2 l}\left(\frac{1}{2}\right)^{k^{\prime}-2 l}\left(\begin{array}{c}
k^{\prime}-2 l \\
i
\end{array}\right) \Phi_{p_{0}}^{i}(\theta) \Phi_{p_{1}}^{k^{\prime}-2 l-i}(\theta) \Phi_{p_{0}}^{l}(\theta) \Phi_{p_{1}}^{l}(\theta) \\
& =\left[\frac{1}{2} \Phi_{p_{0}}(\theta)+\frac{1}{2} \Phi_{p_{1}}(\theta)\right]^{k^{\prime}-2 l} \Phi_{p_{0}}^{l}(\theta) \Phi_{p_{1}}^{l}(\theta) \\
& \leq\left[\frac{1}{2} \Phi_{p_{0}}(\theta)+\frac{1}{2} \Phi_{p_{1}}(\theta)\right]^{k^{\prime}}=\Phi_{p}(\theta)^{k^{\prime}}
\end{aligned}
$$


where the last inequality follows from the AM-GM inequality and the final equality holds because $p=\frac{1}{2}\left(p_{0}+p_{1}\right)$. This yields the lemma because $k^{\prime} \leq k$.

Lemma 7 easily gives the following large deviation inequality.

Corollary 8 Suppose that $p \leq 3 / 4$. Then

$$
\mathbf{P}\left(Z>\frac{7}{8} k \mid \mathcal{F}_{B}\right)<e^{-k / 64}
$$

Proof: We have

$$
\begin{aligned}
\mathbf{E}\left(e^{\theta(Z-p k)} \mid \mathcal{F}_{B}\right) & =e^{-p k \theta} \mathbf{E}\left(e^{\theta Z} \mid \mathcal{F}_{B}\right) \\
& \leq\left[(1-p) e^{-p \theta}+p e^{\theta(1-p)}\right]^{k},
\end{aligned}
$$

by Lemma 7 The quantity inside the square brackets is $\mathbf{E}\left(e^{\theta(Y-p)}\right)$, for a $\operatorname{Bernoulli}(p)$ random variable $Y$. The inequality $\mathbf{E}\left(e^{W}\right) \leq e^{\operatorname{var}(W)}$, valid when $\mathbf{E}(W)=0$ and $W \leq 1$ (see, e.g., 9]), implies that the quantity (54) is at most $\exp \left(\frac{1}{4} \theta^{2} k\right)$ if $\theta \leq 1$. Letting $\theta=\frac{1}{4}$ gives

$$
\mathbf{E}\left(\exp \left[\frac{1}{4}(Z-p k)\right]\right) \leq e^{k / 64}
$$

and hence

$$
\begin{aligned}
\mathbf{P}\left(Z>\frac{7}{8} k \mid \mathcal{F}_{B}\right) & =\mathbf{P}\left(\exp \left[\frac{1}{4}(Z-p k)\right]>\exp \left[\frac{7 k}{32}-\frac{p k}{4}\right] \mid \mathcal{F}_{B}\right) \\
& \leq \exp \left[-\frac{7 k}{32}+\frac{p k}{4}\right] \exp \left[\frac{k}{64}\right]
\end{aligned}
$$

by Markov's inequality. Finally, since $p \leq 3 / 4$, the quantity (57) is at most $e^{-k / 64}$.

The following lemma was used in the proof of Lemma 4 in section 5.

Lemma 9 Fix a set of cards $S$ with $|S| \geq 2^{d-1}$. Then for any set $S^{\prime}$ of vertices of the hypercube we have

$$
\mathbf{P}\left(S \text { does not mix } \mid S \rightarrow_{m} S^{\prime}\right) \leq \alpha^{-d} \frac{1+\lambda(|S|)}{1-\lambda(|S|)} .
$$

Proof: Let $E$ be the event that $S$ does not mix. We have

$$
\begin{aligned}
\mathbf{P}\left(E, S \rightarrow_{m} S^{\prime}\right) & \leq \sum_{k \leq \frac{1}{2}|S|} \sum_{A:|A|=k} \mathbf{P}\left(A \text { avoids } B, S \rightarrow_{m} S^{\prime}\right) \\
& \leq 2^{d-1} \max _{k}\left[2^{d k} \max _{A:|A|=k} \mathbf{P}\left(A \text { avoids } B, S \rightarrow_{m} S^{\prime}\right)\right],
\end{aligned}
$$

where in the summations we write $B$ for $S-A$, the $2^{d-1}$ is an upper bound on the number of $k \leq \frac{1}{2}|S|$, and the $2^{d k}$ is an upper bound on the number of sets $A$ with $|A|=k$. Since $|A| \leq \frac{1}{2}|S|$ and $A \cup B=S$, we must have $|B| \geq \frac{1}{4} 2^{d}$. Hence if $|A|=k$ then

$$
\begin{aligned}
\mathbf{P}\left(A \text { avoids } B, S \rightarrow_{m} S^{\prime}\right) & \leq \sum_{B^{\prime} \subset S^{\prime}} \mathbf{P}\left(A \text { avoids } B, B \rightarrow_{m} B^{\prime}, A \rightarrow_{m} A^{\prime}\right) \\
& \leq \sum_{B^{\prime} \subset S^{\prime}} \mathbf{P}\left(B \rightarrow_{m} B^{\prime}\right) \mathbf{P}\left(A \text { avoids } B \mid B \rightarrow_{m} B^{\prime}\right),
\end{aligned}
$$


where in the summations, we write $A^{\prime}$ for $S^{\prime}-B^{\prime}$. But

$$
\mathbf{P}\left(A \text { avoids } B \mid B \rightarrow_{m} B^{\prime}\right) \leq \sum_{i=0}^{m} \prod_{j=i}^{i+64 c d-1} \mathbf{P}\left(Z(A, B, j)>\frac{7 k}{8} \mid B \rightarrow_{m} B^{\prime}\right) \leq m\left(e^{-k / 64}\right)^{64 c d},
$$

where the last inequality follows from Corollary 8 . Hence

$$
\begin{aligned}
\mathbf{P}\left(A \text { avoids } B, S \rightarrow_{m} S^{\prime}\right) & \leq \sum_{B^{\prime} \subset S^{\prime}} \mathbf{P}\left(B \rightarrow_{m} B^{\prime}\right) m e^{-c k d} \\
& \leq 2^{d k} m e^{-c k d} \max _{B^{\prime}} \mathbf{P}\left(B \rightarrow_{m} B^{\prime}\right)
\end{aligned}
$$

where the $2^{d k}$ is an upper bound on the number of subsets $B^{\prime} \subset S^{\prime}$. But for any $B^{\prime}$ we have

$$
\mathbf{P}\left(B \rightarrow_{m} B^{\prime}\right) \leq \sum_{\hat{S}: \hat{S} \supset B^{\prime}} \mathbf{P}\left(S \rightarrow_{m} \hat{S}\right) \leq 2^{d k}\left(\begin{array}{l}
2^{d} \\
|S|
\end{array}\right)^{-1}(1+\lambda(|S|)) .
$$

It follows that

$$
\begin{aligned}
\mathbf{P}\left(A \text { avoids } B, S \rightarrow_{m} S^{\prime}\right) & \leq 4^{d k} m e^{-c d k}\left(\begin{array}{c}
2^{d} \\
|S|
\end{array}\right)^{-1}(1+\lambda(|S|)) \\
& =\left[4 e^{-c}\right]^{d} \beta \log \alpha c d^{5}\left(\begin{array}{l}
2^{d} \\
|S|
\end{array}\right){ }^{-1}(1+\lambda(|S|)) \\
& \leq \alpha^{-d}\left(\begin{array}{l}
2^{d} \\
|S|
\end{array}\right)
\end{aligned}
$$

where the second inequality follows from the definition of $c$. Finally, since $\mathbf{P}\left(S \rightarrow_{m} S^{\prime}\right) \geq\left(\begin{array}{l}2^{d}{ }_{|S|} \\ )^{-1}\end{array}(1-\right.$ $\lambda(|S|))$, we get $\mathbf{P}\left(A\right.$ avoids $\left.B \mid S \rightarrow_{m} S^{\prime}\right) \leq \alpha^{-d} \frac{1+\lambda(|S|)}{1-\lambda(|S|)}$.

\section{Appendix B}

The purpose of this section is to prove Corollary 13] which is used to bound the root profile. If $K$ is the transition kernel for a Markov chain on the state space $V$, we will consider $K$ as an operator acting on the space of functions $f: V \rightarrow \mathbf{R}$ by

$$
K f(x)=\sum_{y \in V} K(x, y) f(y) .
$$

We will need the following lemma, which was proved by Yuval Peres.

Lemma 10 Let $K$ be a doubly stochastic transition kernel and define $\hat{K}=K K^{t}$. For any function $g: V \rightarrow[0,1]$ and $n \geq 1$ we have

$$
\left\|K^{t} g\right\|_{2}^{2} \leq\langle g, g\rangle^{1-\frac{1}{n}}\left\langle\hat{K}^{n} g, g\right\rangle^{\frac{1}{n}}
$$


Proof: Since $\hat{K}$ is symmetric it is diagonalizable. Thus we can write $g=\sum_{i} \alpha_{i} g^{i}$, where the $g^{i}$ are orthonormal eigenfunctions of $\hat{K}$ with corresponding eigenvalues $\lambda_{i}$. We have

$$
\begin{aligned}
\frac{\left\|K^{t} g\right\|_{2}^{2}}{\langle g, g\rangle} & =\frac{\langle\hat{K} g, g\rangle}{\langle g, g\rangle} \\
& =\frac{\sum_{i} \alpha_{i}^{2} \lambda_{i}}{\sum_{i} \alpha_{i}^{2}} \\
& \leq\left(\frac{\sum_{i} \alpha_{i}^{2} \lambda_{i}^{n}}{\sum_{i} \alpha_{i}^{2}}\right)^{1 / n}=\left(\frac{\left\langle\hat{K}^{n} g, g\right\rangle}{\langle g, g\rangle}\right)^{1 / n},
\end{aligned}
$$

by Jensen's inequality. Multiplying both sides by $\langle g, g\rangle$ yields the lemma.

We will also need the following lemma, which was proved by Keith Ball.

Lemma 11 Let $X$ be a random variable taking values in $[0,1]$ and suppose that $\mathbf{E}(X)=\mu \leq \frac{1}{2}$. Then for any $p>1$ we have

$$
\frac{\mathbf{E}\left(X^{p}\right)}{\mu^{p}}-1 \leq\left(\mu^{1-p}-1\right) \mathbf{E}\left|\frac{X-\mu}{\mu}\right| .
$$

Proof: Let $l=\frac{1}{2} \mathbf{E}(|X-\mu|)$. For a given value of $l$, the l.h.s. of (68) is maximized when $X$ is concentrated on the three values $0, \mu$ and 1 (because it is a convex function of $X$ ). Let $p_{0}, p_{\mu}$ and $p_{1}$ be the respective probabilities. Then $l=p_{1}(1-\mu)=p_{0} \mu$, and hence $p_{\mu}=1-p_{0}-p_{1}=1-\frac{l}{\mu(1-\mu)}$. It follows that

$$
\begin{aligned}
\frac{\mathbf{E}\left(X^{p}\right)}{\mu^{p}}-1 & =\frac{p_{1}+p_{\mu} \mu^{p}}{\mu^{p}}-1 \\
& =l\left[\frac{1}{\mu^{p}(1-\mu)}-\frac{1}{\mu(1-\mu)}\right] \\
& \leq \frac{2 l}{\mu}\left(\mu^{1-p}-1\right),
\end{aligned}
$$

since $1-\mu \geq \frac{1}{2}$, and the proof is complete.

Fix $d_{\star} \leq d$. Recall that the $d_{\star}$-truncated Thorp shuffle is the Markov chain with transition kernel $K_{\star}^{t}=K_{1} \ldots K_{d_{\star}}$. Let $V$ denote the state space of this chain. Corollary 13 is a consequence of the following technical lemma.

Lemma 12 Fix $f: V \rightarrow[0,1]$. Then there is a universal constant $C \in(0,1)$ such that

$$
\left\|K_{\star}^{t} f\right\|_{2}^{2} \leq\|f\|_{1}^{1+1 / C d^{2} d_{\star}^{12}} .
$$

Proof: Suppose that $d_{\star}=1$. Then the truncated Thorp shuffle makes the distribution uniform over $V$ in one step. Thus,

$$
\begin{aligned}
\left\|K_{\star}^{t} f\right\|_{2}^{2} & =\sum_{x \in V}\|f\|_{1}^{2} \frac{1}{|V|} \\
& =\|f\|_{1}^{2} \\
& \leq\|f\|_{1}^{p},
\end{aligned}
$$


for any $p \in[1,2]$, since $\|f\|_{1} \leq 1$. Suppose now that $d_{\star} \geq 2$. Let $c$ be the constant appearing in Corollary [5. We will consider the cases $\|f\|_{1} \leq 6^{-c d_{\star}^{6}}$ and $\|f\|_{1}>6^{-c d_{\star}^{6}}$ separately.

Case 1: $\|f\|_{1} \leq 6^{-c d_{\star}^{6}}$. We show by induction on $d_{\star}$ that $\left\|K_{\star}^{t} f\right\|_{2}^{2} \leq\|f\|_{1}^{1+1 / c d d_{\star}^{5}}$. The base case $d_{\star}=1$ is handled by equation (71) above. Now assume that the result holds for $d_{\star}-1$. Define $\mathcal{L}_{\star}$ as the set of vertices in the cube whose $d_{\star}^{\text {th }}$ coordinate is 0 . Let $\mathcal{B}$ denote the collection of subsets $b$ of $\left\{1, \ldots, 2^{d}\right\}$ such that $X(b)=\mathcal{L}_{\star}$ for some $X \in V$ (i.e., there is a configuration $X \in V$ such that the set of cards occupying $\mathcal{L}_{\star}$ is $\left.b\right)$. For $b \in \mathcal{B}$, define $V_{b}=\left\{X \in V: X(b)=\mathcal{L}_{\star}\right\}$. Let $r=\|f\|_{1}$ and for $\Lambda \subset \mathcal{B}$, define

$$
V_{\Lambda}=\cup_{b \in \Lambda} V_{b}
$$

Let

$$
H=\left\{b \in \mathcal{B}: \frac{\left\|f \mathbf{1}_{V_{b}}\right\|_{1}}{\|f\|_{1}} \geq \frac{r^{-1 / d_{\star}}}{|\mathcal{B}|}\right\}
$$

Since $\sum_{b \in \mathcal{B}} \frac{\left\|f \mathbf{1}_{V_{b}}\right\|_{1}}{\|f\|_{1}}=\frac{\|f\|_{1}}{\|f\|_{1}}=1$, Markov's inequality implies that

$$
\frac{|H|}{|\mathcal{B}|} \leq r^{1 / d_{\star}}
$$

Let $A=V_{H}$ and let $f_{1}=f \mathbf{1}_{A}$ and $f_{2}=f \mathbf{1}_{A^{c}}$. Then

$$
\left\|K_{\star}^{t} f\right\|_{2}^{2}=\left\|K_{\star}^{t} f_{1}+K_{\star}^{t} f_{2}\right\|_{2}^{2} \leq 2\left\|K_{\star}^{t} f_{1}\right\|_{2}^{2}+2\left\|K_{\star}^{t} f_{2}\right\|_{2}^{2} .
$$

We will bound each term on the right hand side separately. First, consider $\left\|K_{\star}^{t} f_{1}\right\|_{2}^{2}$. Let $\hat{K}$ be the transition kernel for the $d_{\star}$-truncated zigzag shuffle, i.e, $\hat{K}=K_{1} \cdots K_{d_{\star}} \cdots K_{1}$. Let $n=$ $c d\left(d_{\star}-1\right)^{4}$. Using Corollary 5 (with $k=1$ ) and combining this with equation (72) gives $\hat{K}^{n}\left(x, V_{H}\right) \leq$ $\exp (\exp (-1)) r^{1 / d_{\star}}$ for all $x$. Hence

$$
\begin{aligned}
\left\langle\hat{K}^{n} f_{1}, \mathbf{1}_{A}\right\rangle & =|V|^{-1} \sum_{x} f_{1}(x) \hat{K}^{n}\left(x, V_{H}\right) \\
& \leq\left\|f_{1}\right\|_{1} \exp (\exp (-1)) r^{1 / d_{\star}} .
\end{aligned}
$$

Finally, Lemma 10 gives

$$
\begin{aligned}
\left\|K_{\star}^{t} f_{1}\right\|_{2}^{2} & \leq\left\langle f_{1}, f_{1}\right\rangle^{1-1 / n}\left\langle\hat{K}^{n} f_{1}, f_{1}\right\rangle^{1 / n} \\
& \leq\left\langle f_{1}, f_{1}\right\rangle^{1-1 / n}\left\langle\hat{K}^{n} f_{1}, \mathbf{1}_{A}\right\rangle^{1 / n}
\end{aligned}
$$

where the second inequality holds because $f_{1} \leq \mathbf{1}_{A}$. Putting this all together, we get

$$
\begin{aligned}
\left\|K_{\star}^{t} f_{1}\right\|_{2}^{2} & \leq\left\langle f_{1}, f_{1}\right\rangle^{1-1 / n}\left[\left\|f_{1}\right\|_{1}(\exp (\exp (-1))) r^{1 / d_{\star}}\right]^{1 / n} \\
& \leq 2\left(\frac{\left\langle f_{1}, f_{1}\right\rangle}{\left\|f_{1}\right\|_{1}}\right)^{1-1 / n} \times\left\|f_{1}\right\|_{1} \times r^{1 / d_{\star} n}
\end{aligned}
$$

since $\exp \left(\frac{1}{n} \exp (-1)\right) \leq 2$ for all $n$. Since $n=c d\left(d_{\star}-1\right)^{4}$, and $\frac{\left\langle f_{1}, f_{1}\right\rangle}{\left\|f_{1}\right\|_{1}} \leq 1$, we have

$$
\left\|K_{\star}^{t} f_{1}\right\|_{2}^{2} \leq 2 r^{1 / c d d_{\star}\left(d_{\star}-1\right)^{4}}\|f\|_{1} .
$$


Next we bound $\left\|K_{\star}^{t} f_{2}\right\|_{2}^{2}$. Since $K_{d_{\star}}$ is symmetric it contracts $l^{2}$. Hence

$$
\begin{aligned}
\left\|K_{\star}^{t} f_{2}\right\|_{2}^{2} & \leq\left\|K_{\left(d_{\star}-1\right)} \cdots K_{1} f_{2}\right\|_{2}^{2} \\
& =\sum_{b \in \mathcal{B}}\left\|K_{\left(d_{\star}-1\right)} \cdots K_{1} f_{2} \mathbf{1}_{V_{b}}\right\|_{2}^{2} .
\end{aligned}
$$

Note that $K_{1} \cdots K_{\left(d_{\star}-1\right)}$ is just the transition kernel for a $\left(d_{\star}-1\right)$-truncated Thorp shuffle and that the $V_{b}$ are communicating classes for this process. Thus, we can use the induction hypothesis to bound each $\left\|K_{\left(d_{\star}-1\right)} \cdots K_{1} f_{2} \mathbf{1}_{V_{b}}\right\|_{2}^{2}$, provided that the corresponding normalized $l_{1}$ norm $\frac{\left\|f_{2} \mathbf{1}_{V_{b}}\right\|_{1}}{\left\|\mathbf{1}_{V_{b}}\right\|_{1}}$ is sufficiently small. Define $r_{b}:=\frac{\left\|f_{2} \mathbf{1}_{V_{b}}\right\|_{1}}{\left\|\mathbf{1}_{V_{b}}\right\|_{1}}$. We claim that for every $b \in \mathcal{B}$ we have $r_{b} \leq r^{\frac{d_{\star}-1}{d_{\star}}}$. To see this, note that if $b \in H$, then $\left\|f_{2} \mathbf{1}_{V_{b}}\right\|_{1}=0$ and the claim holds trivially, so assume $b \notin H$. Then

$$
\begin{aligned}
\frac{\left\|f_{2} \mathbf{1}_{V_{b}}\right\|_{1}}{\left\|\mathbf{1}_{V_{b}}\right\|_{1}} & =\left\|f_{2} \mathbf{1}_{V_{b}}\right\|_{1}|\mathcal{B}| \\
& \leq\left\|f \mathbf{1}_{V_{b}}\right\|_{1}|\mathcal{B}| \\
& \leq r^{\frac{-1}{d_{\star}}}\|f\|_{1}=r^{\frac{d_{\star}-1}{d_{\star}}}
\end{aligned}
$$

where the first equality holds because $\left\|\mathbf{1}_{V_{b}}\right\|_{1}=|\mathcal{B}|^{-1}$, the second inequality holds because $b \notin H$ (and by the definition of $H$ ) and last equality holds because $\|f\|_{1}=r$. It follows that

$$
r_{b} \leq r^{\frac{d_{\star}-1}{d_{\star}}} \leq 6^{-c\left(d_{\star}-1\right) d_{\star}{ }^{5}} \leq 6^{-c\left(d_{\star}-1\right)^{6}} .
$$

Thus we can apply the induction hypothesis, which gives

$$
\begin{aligned}
\left\|K_{\left(d^{\prime}-1\right)} \cdots K_{1} f_{2} \mathbf{1}_{V_{b}}\right\|_{2}^{2} & \leq r_{b}^{1 / c d\left(d_{\star}-1\right)^{5}}\left\|f_{2} \mathbf{1}_{V_{b}}\right\|_{1} \\
& \leq r^{1 / c d d_{\star}\left(d_{\star}-1\right)^{4}}\left\|f_{2} \mathbf{1}_{V_{b}}\right\|_{1},
\end{aligned}
$$

where the second inequality follows from the first inequality in (86). Combining this with equation (82) and using the fact that $f_{2} \leq f$ gives

$$
\left\|K_{\star}^{t} f_{2}\right\|_{2}^{2} \leq r^{1 / c d d_{\star}\left(d_{\star}-1\right)^{4}}\|f\|_{1} .
$$

We are now ready to bound $\left\|K^{t} f\right\|_{2}^{2}$. Combining equations (89), (80) and (73), we get

$$
\left\|K_{\star}^{t} f\right\|_{2}^{2} \leq\left(6 r^{1 / c d d_{\star}\left(d_{\star}-1\right)^{4}}\right)\|f\|_{1} .
$$

Since $(k-1)^{-4}-k^{-4} \geq k^{-5}$ for integers $k \geq 2$, the quantity (90) is at most

$$
6 r^{1 / c d d_{\star}^{5}+1 / c d d_{\star}^{6}}\|f\|_{1} \leq r^{1 / c d d_{\star}^{5}}\|f\|_{1},
$$

since $r \leq 6^{-c d d_{\star}^{6}}$. This concludes the proof in the case $r \leq 6^{-c d d_{\star}^{6}}$.

Case 2: $r>6^{-c d d_{\star}^{6}}$. Let $C$ be an integer that is larger than $2^{15} c^{2} 15 \log 2 \log 6$. We will show by induction on $d_{\star}$ that

$$
\left\|K_{\star}^{t} f\right\|_{2}^{2} \leq r^{1+1 / C d^{2} d_{\star}^{12}} .
$$

The base case $d_{\star}=1$ was handled earlier by equation (71). 
Now, fix $d_{\star} \geq 2$ and $f: V \rightarrow[0,1]$ and suppose that $r=\|f\|_{1}>6^{-c d d_{\star}^{6}}$. We can assume w.l.o.g. that $r \leq \frac{1}{2}$. Otherwise, let $h=1-f$, and suppose that the result holds for $h$, i.e., for $q=1 / C d^{2} d_{\star}^{12}$ we have

$$
\left\|K_{\star}^{t} h\right\|_{2}^{2} \leq\|h\|_{1}^{1+q}
$$

or equivalently,

$$
\|h\|_{1}-\left\|K_{\star}^{t} h\right\|_{2}^{2} \geq\left[1-\|h\|_{1}^{q}\right]\|h\|_{1} .
$$

Note that

$$
\begin{aligned}
\left\|K_{\star}^{t} h\right\|_{2}^{2} & =\left\langle K_{\star}^{t}(1-f), K_{\star}^{t}(1-f)\right\rangle \\
& =\left\langle K_{\star}^{t} \mathbf{1}, K_{\star}^{t} \mathbf{1}\right\rangle-2\left\langle K_{\star}^{t} \mathbf{1}, K_{\star}^{t} f\right\rangle+\left\langle K_{\star}^{t} f, K_{\star}^{t} f\right\rangle \\
& =1-2\|f\|_{1}+\left\|K_{\star}^{t} f\right\|_{2}^{2} \\
& =\|h\|_{1}-\|f\|_{1}+\left\|K_{\star}^{t} f\right\|_{2}^{2},
\end{aligned}
$$

where the third equality holds because $K_{\star}^{t}$ is doubly stochastic and hence $K_{\star}^{t} \mathbf{1}=\mathbf{1}$. Thus

$$
\|h\|_{1}-\left\|K^{t} h\right\|_{2}^{2}=\|f\|_{1}-\left\|K^{t} f\right\|_{2}^{2} .
$$

Define $u:[0,1] \rightarrow \mathbf{R}$ by

$$
u(x)=\left(1-x^{q}\right) x=\frac{x(1-x)}{1+x^{q}+\cdots+x^{1-q}},
$$

so the RHS of (91) is $u\left(\|h\|_{1}\right)$. Since the numerator on the RHS of (97) is symmetric about $\frac{1}{2}$ and the denominator is increasing, we have $u(x) \geq u(1-x)$ if $x \leq \frac{1}{2}$. This, combined with equation (96)), shows that equation (91) is still true if we replace the $h$ by $f$. Thus we can assume henceforth that $r \leq \frac{1}{2}$.

Let $\mathcal{B}$ and $V_{b}$ be as defined above. Then

$$
\begin{aligned}
\left\|K_{\star}^{t} f\right\|_{2}^{2} & \leq\left\|K_{\left(d_{\star}-1\right)} \cdots K_{1} f\right\|_{2}^{2} \\
& =\sum_{b \in \mathcal{B}}\left\|K_{\left(d_{\star}-1\right)} \cdots K_{1} f \mathbf{1}_{V_{b}}\right\|_{2}^{2} .
\end{aligned}
$$

For $b \in \mathcal{B}$, define $r_{b}=\frac{\left\|f \mathbf{1}_{V_{b}}\right\|_{1}}{\left\|\mathbf{1}_{V_{b}}\right\|_{1}}=\left\|f \mathbf{1}_{V_{b}}\right\|_{1}|\mathcal{B}|$. We may assume that

$$
\left\|K_{\left(d_{\star}-1\right)} \cdots K_{1} f \mathbf{1}_{V_{b}}\right\|_{2}^{2} \leq r_{b}^{1 / C d^{2}\left(d_{\star}-1\right)^{12}}\left\|f \mathbf{1}_{V_{b}}\right\|_{1} .
$$

(In the case where $r_{b} \leq 6^{-c d\left(d_{\star}-1\right)^{6}}$ this was proved earlier, since $c d\left(d_{\star}-1\right)^{5} \leq C d^{2}\left(d_{\star}-1\right)^{12}$; in the case where $r_{b}>6^{-c} d\left(d_{\star}-1\right)^{6}$ this is the induction hypothesis.) Combining this with (99) gives

$$
\left\|K_{\star}^{t} f\right\|_{2}^{2} \leq \sum_{b \in \mathcal{B}} r_{b}^{1 / C d^{2}\left(d_{\star}-1\right)^{12}}\left\|f \mathbf{1}_{V_{b}}\right\|_{1}=|\mathcal{B}|^{-1} \sum_{b \in \mathcal{B}} r_{b}^{1+1 / C d^{2}\left(d_{\star}-1\right)^{12}} .
$$

Thus, unless

$$
|\mathcal{B}|^{-1} \sum_{b \in \mathcal{B}} r_{b}^{1+1 / C d^{2}\left(d_{\star}-1\right)^{12}} \geq r^{1+1 / C d^{2} d_{\star}^{12}}
$$


the result is immediate. So assume that (100) holds. For $b \in \mathcal{B}$, define $w_{b}=\frac{\left\|f \mathbf{1}_{V_{b}}\right\|_{1}}{\|f\|_{1}}$. Note that $\sum_{b \in \mathcal{B}} w_{b}=1$. Let $U$ be chosen uniformly at random from $\mathcal{B}$. Let $p=1+1 / C d^{2}\left(d_{\star}-1\right)^{12}$. Dividing both sides of (100) by $r^{p}$ gives

$$
\frac{\mathbf{E}\left(r_{U}^{p}\right)}{r^{p}} \geq r^{1 / C d^{2} d_{\star}^{12}-1 / C d^{2}\left(d_{\star}-1\right)^{12}} .
$$

Using the inequality $k^{-12}-(k-1)^{-12} \leq-k^{-13}$, valid for integers $k \geq 2$, and subtracting 1 from both sides of (101) gives

$$
\frac{\mathbf{E}\left(r_{U}^{p}\right)}{r^{p}}-1 \geq r^{-1 / C d^{2} d_{\star}^{13}}-1
$$

Let $\pi$ be the uniform probability measure on $\mathcal{B}$ and let $\nu$ be the measure on $\mathcal{B}$ defined by the $w_{b}$. Define

$$
\|\pi-\nu\|_{T V}=\left.\frac{1}{2} \sum_{b \in \mathcal{B}}\left|w_{b}-\right| \mathcal{B}\right|^{-1}\left|=\frac{1}{2} \mathbf{E}\right| \frac{r_{U}-r}{r} \mid .
$$

Note that $\mathbf{E}\left(r_{U}\right)=|\mathcal{B}|^{-1} \sum_{b \in \mathcal{B}} r_{b}=r$. Plugging $X=r_{U}$ and $\mu=r$ into Lemma 11] and combining with equation (102) gives

$$
\begin{aligned}
2\|\pi-\nu\|_{T V} & \geq \frac{r^{-1 / C d^{2} d_{\star}^{13}}-1}{r^{-1 / C d^{2}\left(d_{\star}-1\right)^{12}}-1} \\
& =\frac{\exp \left(\frac{-\log r}{C d^{2} d_{\star}^{13}}\right)-1}{\exp \left(\frac{-\log r}{C d^{2}\left(d_{\star}-1\right)^{12}}\right)-1} .
\end{aligned}
$$

Since $r>6^{-c d d_{\star}^{6}}$, the quantities in the exponents in (104) are in $\left(0, \frac{1}{2}\right]$. (Recall that $C$ is much larger than $c$.) Hence, the fact that $\frac{e^{t}-1}{t} \in[1,2]$ whenever $t \in\left(0, \frac{1}{2}\right]$ implies that the quantity in (104) is at least

$$
\frac{\left(d_{\star}-1\right)^{12}}{2 d_{\star}^{13}} \geq \frac{d_{\star}^{-1}}{2^{13}}
$$

where the inequality holds because $d_{\star} \geq 2$ and hence $\frac{d_{\star}-1}{d_{\star}} \geq \frac{1}{2}$. It follows that $\|\pi-\nu\|_{T V} \geq 2^{-14} d_{\star}^{-1}$. Note that

$$
\begin{aligned}
2\|\pi-\nu\|_{T V} & =\sum_{b \in \mathcal{B}} \max (\nu(b), \pi(b))-\min (\nu(b), \pi(b)) ; \\
2 & =\sum_{b \in \mathcal{B}} \max (\nu(b), \pi(b))+\min (\nu(b), \pi(b)) .
\end{aligned}
$$

Subtracting the first equation from the second and dividing by 2 gives

$$
1-\|\pi-\nu\|_{T V}=\sum_{b \in \mathcal{B}} \min (\nu(b), \pi(b)) .
$$

Recall that $\hat{K}$ is the transition kernel for the $d_{\star}$-truncated zigzag shuffle. Note that

$$
\begin{aligned}
\left\langle f, \hat{K}^{n} f\right\rangle & =\sum_{b \in \mathcal{B}}\left\langle f \mathbf{1}_{V_{b}},\left(\hat{K}^{n} f\right) \mathbf{1}_{V_{b}}\right\rangle \\
& \leq \sum_{b \in \mathcal{B}} \min \left(\left\|f \mathbf{1}_{V_{b}}\right\|_{1},\left\|\left(\hat{K}^{n} f\right) \mathbf{1}_{V_{b}}\right\|_{1}\right) \\
& =\|f\|_{1} \sum_{b \in \mathcal{B}} \min \left(w_{b}, \frac{\left\|\left(\hat{K}^{n} f\right) \mathbf{1}_{V_{b}}\right\|_{1}}{\|f\|_{1}}\right),
\end{aligned}
$$


where the inequality holds because $f \mathbf{1}_{V_{b}} \leq 1$ and $\left(\hat{K}^{n} f\right) \mathbf{1}_{V_{b}} \leq 1$. Let $n=15 c d d_{\star}^{5} \log 2$. Corollary 5 implies that $\frac{\left\|\left(\hat{K}^{n} f\right) \mathbf{1}_{V_{b}}\right\|_{1}}{\|f\|_{1}} \leq \alpha\left(d_{\star}\right)|\mathcal{B}|^{-1}$, where $\alpha(k):=\exp \left(2^{-15 k}\right)$. Hence,

$$
\begin{aligned}
\left\langle f, \hat{K}^{n} f\right\rangle & \leq\|f\|_{1} \alpha\left(d_{\star}\right) \sum_{b \in \mathcal{B}} \min \left(w_{b}, \frac{1}{|\mathcal{B}|}\right) \\
& =\|f\|_{1} \alpha\left(d_{\star}\right)\left(1-\|\nu-\pi\|_{T V}\right) \\
& \leq\|f\|_{1} \alpha\left(d_{\star}\right)\left[1-2^{-14} d_{\star}^{-1}\right] .
\end{aligned}
$$

Hence Lemma 10 gives

$$
\begin{aligned}
\left\|K_{\star}^{t} f\right\|_{2}^{2} & \leq\langle f, f\rangle^{1-1 / n}\left[\|f\|_{1} \alpha\left(d_{\star}\right)\left(1-2^{-14} d_{\star}^{-1}\right)\right]^{1 / n} \\
& =\left(\frac{\langle f, f\rangle}{\|f\|_{1}}\right)^{1-1 / n} \times\|f\|_{1} \times \alpha\left(d_{\star}\right)^{1 / n} \times\left(1-2^{-14} d_{\star}^{-1}\right)^{1 / n} \\
& \leq\|f\|_{1} \exp \left(\frac{1}{n}\left[2^{-15 d_{\star}}-d_{\star}^{-1} 2^{-14}\right]\right) \\
& \leq\|f\|_{1} \exp \left(-1 / 2^{15} c d d_{\star}^{6} 15 \log 2\right),
\end{aligned}
$$

since $\frac{\langle f, f\rangle}{\|f\|_{1}} \leq 1$ and $2^{-15 k} \leq 2^{-15} k^{-1}$ for all positive integers $k$. Finally, since $r>6^{-c d d_{\star}^{6}}=$ $\exp \left(-c d d_{\star}^{6} \log 6\right)$, we have $r^{1 / C d^{2} d_{\star}^{12}} \geq \exp \left(-1 / 2^{15} c d d_{\star}^{6} 15 \log 2\right)$. (Recall that $C>2{ }^{15} c^{2} 15 \log 2 \log 6$.) It follows that $\left\|K_{\star}^{t} f\right\|_{2}^{2} \leq r^{1 / C d^{2} d_{\star}^{12}}\|f\|_{1}$. This completes the proof.

To bound the root profile, we actually used the following corollary.

Corollary 13 Fix $S \subset V$ and let

$$
x=\frac{|S|}{\left(2^{d}\right) !}
$$

Let $\{p(x, y)\}$ be the transition probabilities for a round of the Thorp shuffle. Then there is a universal constant $C>0$ such that

$$
\|p(S, \cdot)\|_{2}^{2} \leq x^{1+C / d^{14}}
$$

Proof: Let $f=\mathbf{1}_{S}$ and $d_{\star}=d$ and apply Lemma 12, (Note that if $K$ is the transition kernel for a round of the Thorp shuffle then $p(S, \cdot)=K^{t} f$.)

Acknowledgments. I am grateful to K. Ball, T. Coulhon, E. Mossel, C. Nair, Y. Peres, A. Sinclair, D. Wilson, P. Winkler and J. Zuniga for invaluable discussions.

I want to thank Jessica Zuniga for pointing out an error in the conference version of this paper. Yuval Peres proved Lemma 10] and Keith Ball proved Lemma 11].

I also want to thank Christian Borgs and Jennifer Chayes for giving me the opportunity to spend the year at the Theory Group of Microsoft Research, where I did much of this research. 


\section{References}

[1] Bayer, D. and Diaconis, P. Tracing the dovetail shuffle to its lair, Annals of Applied Probability. 2 (1992). pp. 294-313.

[2] Çam, H. Rearrangeability of $(2 n-1)$-Stage Shuffle-Exchange Networks. SIAM Journal on Computing. 32 (2003). pp. 557-585.

[3] Diaconis, P. Personal Communication.

[4] Diaconis, P. Group representations in Probability and Statistics, volume 11 of Lecture NotesMonograph series. Institute of Mathematical Statistics, Hayward, California, 1988.

[5] Diaconis, P. and Fill, J. Strong stationary times via a new form of duality. Annals of Probability 18 (1990). pp. 1483-1522.

[6] Knuth, D. Searching and Sorting, The Art of Computer Programming III. Addison-Wesley, 1973.

[7] Morris, B. The mixing time for simple exclusion. To appear in Annals of Applied Probability.

[8] Morris, B. and Peres, Y. Evolving sets, mixing and heat kernel bounds. To appear in Probability Theory and Related Fields. Availible at http://front.math.ucdavis.edu/math.PR/0305349.

[9] Steiger, W. A best possible Kolmogoroff-type inequality for martingales and a characteristic propery, Annals of Mathematical Statistics. 40 (1969). pp. 764-769.

[10] Thorp, E. Nonrandom shuffling with applications to the game of Faro, Journal of the American Statistical Association. 68 (1973). pp. 842-847. 